\title{
Should COVID-19 testing programs also screen for HIV?
}

— Cite as: CMAJ 2021 May 10;193:E702-3. doi: 10.1503/cmaj.1095940

Posted on cmajnews.com on April 27, 2021

$\mathrm{T}$ he COVID-19 pandemic may be facilitating the spread of HIV, but combined screening programs could reduce the spread of both viruses, according to recent research.

Worst-case estimates by North American researchers suggest that disruptions in HIV prevention and care during the pandemic may result in a $9 \%$ increase in new HIV infections over the next few years.

In Chicago, HIV screening sites reported an overall $49 \%$ drop in testing during the "chaos" of the first months of the pandemic, said Dr. Kimberly Stanford, an emergency physician at the University of Chicago Medicine (UCM). "Everything that wasn't essential medical care kind of fell by the wayside."

However, by incorporating HIV phlebotomy into a rapid COVID-19 testing program, UCM's emergency department was able to maintain prepandemic screening volumes, performing more than 14200 HIV screens in 2020, up from 11861 in 2019. The combined screening program did not require extra personnel and the hospital's HIV care program handled test review and follow up.

"Not only were we finding more HIV... the proportion of our diagnoses that were acute HIV was much higher [than in previous years]," said Stanford who led a study of the program published in JAMA Internal Medicine.

Acute HIV infections accounted for 12 of 46 new HIV diagnoses at UCM during the pandemic - a significant increase compared to the past four years, according to Stanford and her colleagues. Meanwhile, other local emergency departments saw a $25 \%$ drop in diagnoses of acute HIV infection, although this was not a statistically significant reduction.

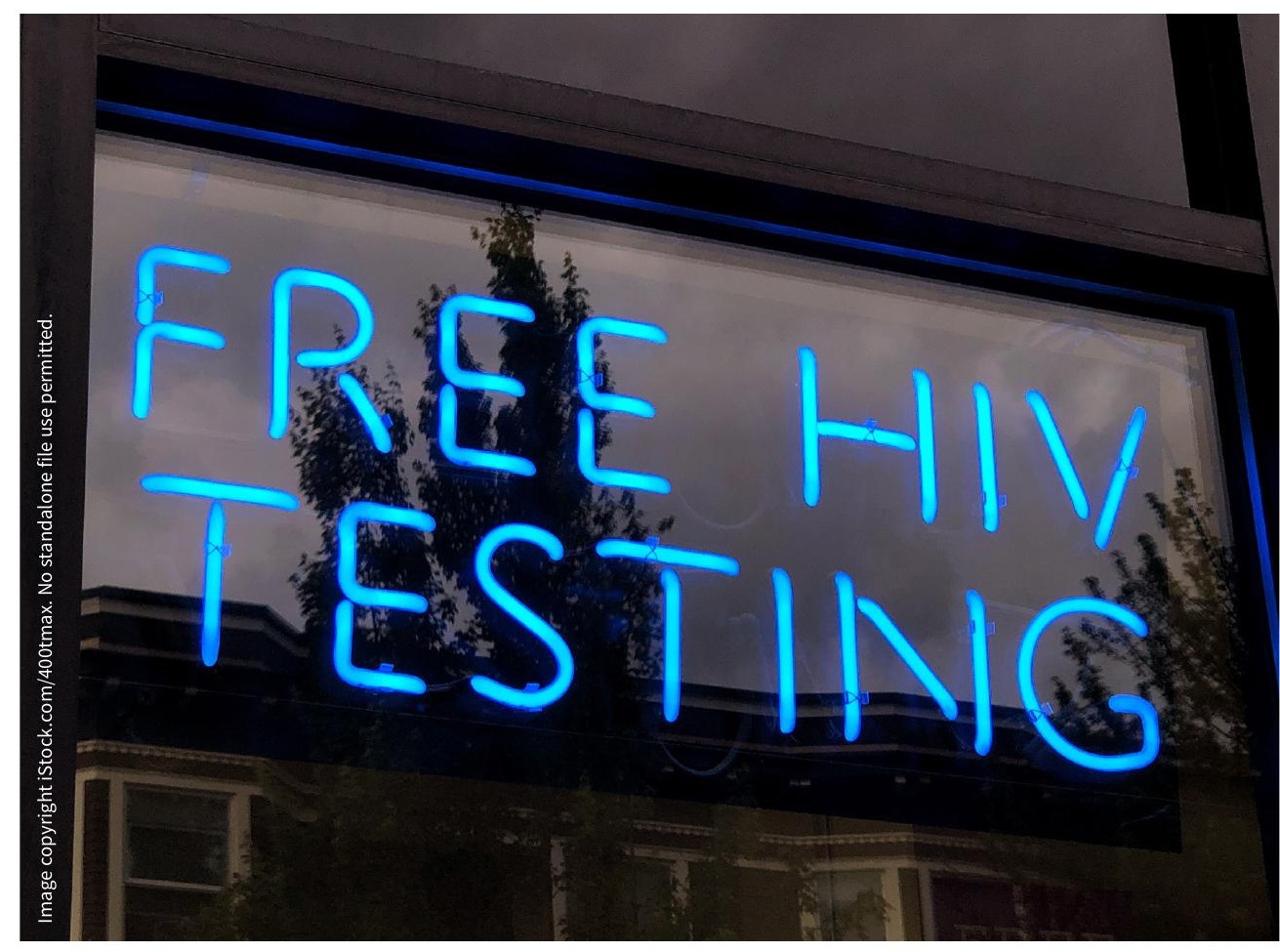

The pandemic has disrupted HIV prevention and care but may present an opportunity to boost screening.

Detecting HIV during the acute stage when viral loads are highest is "extremely important," said Stanford. "You are the most contagious that you will be throughout the entire time that you have HIV."

Earlier treatment also leads to better outcomes for people with HIV, as well as reducing their chances of infecting others, "which is huge from a public health standpoint," she said.

According to Stanford and coauthors, the uptick in diagnoses of acute HIV infection at UCM may be linked to increased screening, more people seeking care for the flu-like symptoms of acute HIV because of concern about COVID-19, or increased transmissions due to pandemic disruptions in HIV prevention. As such, "HIV screening programs, particularly in emergency departments, should incorporate or even link HIV screening to COVID-19 testing."

These findings echo those of an earlier modelling study by American and Canadian researchers that estimated that combined testing for HIV and SARS-CoV-2 had the potential to reduce new HIV infections by up to $17 \%$ over five years - so long as $90 \%$ of people are offered the tests and about $60 \%$ accept. That study estimated that a scaled-up program would cost US\$20-220 million. However, the authors argued the investment would be "costsaving in the long-term." 
Canada has not released data on how the COVID-19 pandemic has affected HIV screening and care. However, Dr. Ameeta Singh said she is "absolutely certain testing rates have declined."

"I would not be at all surprised if testing overall for sexually transmitted and blood-borne infections has gone down," said Singh, a clinical professor of medicine at the University of Calgary. She noted that it's tough getting anyone to undergo HIV testing during the pandemic - even people who are taking pre-exposure prophylaxis because of their higher risk for HIV. "They don't want to go to the lab."

Social distancing policies limit the number of people allowed inside HIV testing sites, and outreach teams that once offered testing at various community organizations have stopped doing so, Singh said. Appointments may be scarce or require advance booking, which presents a barrier for some patients. And, although Canada recently approved the sale of self-testing kits for HIV, the cost (about \$35) may be another barrier.

Singh said incorporating HIV and COVID-19 testing would make sense in Canada, especially in areas with populations at higher risk of HIV.

Dr. Nitika Pant Pai, an associate professor of medicine at McGill University, said people who are marginalized, displaced or unable to socially distance would benefit from such a strategy.

Combining screening for HIV and COVID-19 could also help Canada reach its goal of diagnosing 95\% of all people living with HIV, treating $95 \%$ of all those diagnosed, and achieving viral suppression among $95 \%$ of those treated.

Even before the pandemic, Canada fell short of these targets, and pandemic disruptions will likely continue until the end of the year, Pai said. But given the opportunity, "many affected by HIV would like to be tested if they suspect exposure."

\section{Diana Duong, CMAJ}

Content licence: This is an Open Access article distributed in accordance with the terms of the Creative Commons Attribution (CC BY-NC-ND 4.0) licence, which permits use, distribution and reproduction in any medium, provided that the original publication is properly cited, the use is noncommercial (i.e., research or educational use), and no modifications or adaptations are made. See: https://creativecommons.org/ licenses/by-nc-nd/4.0/ 